\title{
Acute Disruption of Cytochrome Oxidase Activity in Brain in a Perinatal Rat Stroke Model
}

\author{
CARRIE NELSON AND FAYE S. SILVERSTEIN \\ Departments of Pediatrics and Neurology, University of Michigan, Ann Arbor, \\ Michigan 48109-0570
}

\section{ABSTRACT}

Cytochrome oxidase histochemistry in frozen brain sections provides an estimate of mitochondrial respiratory function and neuronal metabolic activity. We hypothesized that in a perinatal rodent stroke model acute selective disruption of cytochrome oxidase activity could identify neuronal populations susceptible to irreversible ischemic injury. In 7-d-old rats, focal ischemic injury was induced by right carotid ligation followed by $8 \% \mathrm{O}_{2}$ exposure $(2.75 \mathrm{~h})$; this procedure elicits ipsilateral forebrain injury with prominent hippocampal lesions, often including dentate gyrus. Cytochrome oxidase activity was quantitated by densitometry in brain sections prepared from animals killed $1 \mathrm{~h}(n=$ $8), 24 \mathrm{~h}(n=11)$, or $5 \mathrm{~d}(n=3)$ posthypoxia. At $1 \mathrm{~h}$ posthypoxia, in six of eight there was diffuse ipsilateral suppression of cytochrome oxidase activity $(p=0.0001$, two-way analysis of variance, compared with values in contralateral hemisphere), which was most marked in periventricular regions including dentate gyrus $(-29 \%)$, medial habenula $(-38 \%)$, and posterolateral thalamic nucleus $(-42 \%)$. Nissl staining of adjacent sections was completely intact in four of eight, and four of eight had subtle focal reductions in staining. At $24 \mathrm{~h}$ and $5 \mathrm{~d}$ posthypoxia,

Mitochondrial dysfunction seems to play a pivotal role in the evolution of ischemic neuronal injury (1-4). Pathogenetically relevant sequelae of ischemia-induced mitochondrial injury include decreased ATP production, increased intracellular calcium accumulation and liberation of arachidonic acid and related toxic metabolites. CytOx is the terminal complex of the mitochondrial respiratory chain that generates ATP via oxidative phosphorylation. Studies in adult brain have reported acute, ischemiainduced suppression of CytOx activity, corresponding

Received September 28, 1993; accepted March 7, 1994.

Correspondence: Dr. F. S. Silverstein, Room 6028, Kresge II Building, Box 0570, University of Michigan, Ann Arbor, MI 48109-0570.

Supported by grants from the American Heart Association of Michigan, the American Heart Association, and USPHS Grant NS 26142.

${ }^{1}$ Preliminary reports of these data were presented at the annual meetings of the Child Neurology Society, Portland, OR, October 1991; the Society for Neuroscience, New Orleans, LA, November 1991; and the Princeton Conference on Cerebrovascular Disease, Rochester, MI, May 1992. heterogeneous suppression of cytochrome oxidase activity persisted in the lesioned hemisphere, with close anatomic correspondence between loss of cytochrome oxidase activity and loss of Nissl staining. Additional studies indicated that the threshold duration of $8 \% \mathrm{O}_{2}$ exposure (after carotid ligation) for suppression of cytochrome oxidase activity was 1-2 h. Disruption of cytochrome oxidase activity represents one of the earliest detectable indicators of neuronal injury in this perinatal stroke model. The threshold duration of hypoxia to suppress its activity preceded or coincided with that to elicit injury, and the prominent loss of activity in dentate gyrus paralleled its enhanced susceptibility to ischemic injury at this developmental stage. (Pediatr Res 36: 12-19, 1994)

\author{
Abbreviations \\ Cytox, cytochrome oxidase \\ PND, postnatal day \\ CA, cornu Ammonis \\ ANOVA, analysis of variance \\ NMDA, N-methyl-D-aspartate
}

with the distribution of irreversible injury (5) and the onset of neurologic deficits (6).

Histochemical assays of CytOx activity in frozen brain sections seem to provide reasonable estimates of neuronal mitochondrial respiratory function and antecedent in vivo neuronal metabolic activity. The intensity of staining corresponds closely with the amount of enzyme detected immunochemically and with CytOx activity measured spectrophotometrically $(7,8)$; thus, optical densitometry can be used to estimate enzyme activity (9). Functional neuronal compartments, regional differences in metabolic activity within brain, and experimentally induced changes in neuronal activity have been delineated with this method (7-9). The first histochemical study of ischemia-induced changes in CytOx activity in adult brain reported extensive reductions after $30 \mathrm{~min}$ of reperfusion; no subsequent time intervals were assessed (5). 
Several pathophysiologic features of ischemic injury in perinatal rodent stroke models differ from patterns observed in adult experimental animals. In hippocampus, the selective delayed injury to CA1 pyramidal neurons characteristic of adult lesions is not seen and pyramidal cell loss is more diffuse (10); also, the dentate gyrus (rather than the hilus) is vulnerable $(11,12)$. Maturity of synaptic circuitry is likely a critical determinant for some of the major age-related differences in outcome; to what extent developmental differences in oxidative metabolism contribute is uncertain.

We hypothesized that in a perinatal rodent stroke model loss of CytOx activity would be a sensitive, early indicator of neuronal injury. We studied a wellcharacterized perinatal rodent stroke model elicited by unilateral carotid ligation and subsequent exposure to $8 \%$ $\mathrm{O}_{2}$ in PND 7 rats (10). The severity of injury, which is related to the duration of hypoxic exposure, is somewhat variable; after $2.5-3 \mathrm{~h}$ of exposure to $8 \% \mathrm{O}_{2}$, prominent ipsilateral forebrain injury evolves in the majority of lesioned animals (13-16). The contribution of disruption of energy metabolism to the evolution of injury in this model has been extensively evaluated; high-energy phosphates are depleted in the ischemic hemisphere during hypoxia and subsequently recover incompletely $(15,17)$. In this study, we compared CytOx activity bilaterally in nine brain regions of PND 7 rats that underwent carotid ligation followed by $8 \% \mathrm{O}_{2}$ exposure $(2.75 \mathrm{~h})$ and were killed 1 or $24 \mathrm{~h}$ later, and in additional animals exposed to $0-2 \mathrm{~h}$ of $8 \% \mathrm{O}_{2}$, to determine the relationship between duration of hypoxic exposure and loss of CytOx activity.

\section{METHODS}

Animal methods. Experiments were done in PND 7 unsexed Sprague-Dawley rats. With the rats under ether anesthesia, the right carotid artery was ligated, as previously described $(10,13)$. After a 1- to 2-h recovery period with their dams, animals were exposed to $8 \% \mathrm{O}_{2}$, balance $\mathrm{N}_{2}(0-165 \mathrm{~min})$, in glass chambers set in warm $\left(36.5^{\circ} \mathrm{C}\right)$ water baths. The surgical protocol was approved by the University of Michigan Animal Care and Use Committee. Animals were killed by decapitation, and their brains were rapidly removed, frozen in dry ice, and stored at $-70^{\circ} \mathrm{C}$.

The extent of injury resulting from this procedure can be readily estimated by PND 12 . Slight variations in the experimental protocol used (with respect to anesthetic, postsurgery recovery period, ambient temperature, and duration of hypoxia) influence the severity of injury. Ligation alone does not result in tissue injury. In our laboratory, after ligation, exposure to $8 \% \mathrm{O}_{2}$ for $2.5 \mathrm{~h}$ results in readily quantifiable ipsilateral forebrain injury in about two thirds of the animals $(>10 \%$ reduction in hemisphere mass by PND 12) (14). In animals exposed to $8 \% \mathrm{O}_{2}$ for $1 \mathrm{~h}$, no lesions are detected; the threshold duration that results in histopathologic injury in a substantial fraction of animals is between 60 and $120 \mathrm{~min}$
(13). Under the conditions used for these experiments, with a maximum duration of $8 \% \mathrm{O}_{2}$ exposure of $2.75 \mathrm{~h}$, the most common outcome is mild-to-moderate ipsilateral forebrain ischemic injury; massive infarction (which can be elicited by longer duration of exposure to $8 \% \mathrm{O}_{2}$ ) is uncommon.

Histology. Serial 20- $\mu \mathrm{m}$ frozen sections were prepared for CytOx assays and Nissl staining. Preliminary experiments were done to optimize the CytOx assay in PND 7-8 rat brain [using methods developed by Hevner and Wong-Riley (7)]. Sections were incubated in a $0.1 \mathrm{M}$

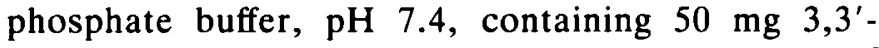
diaminobenzidine, $25 \mathrm{mg}$ of cytochrome $c, 20 \mathrm{mg}$ of catalase, and $4 \mathrm{~g}$ of sucrose $/ 100 \mathrm{~mL}$ for $1-4 \mathrm{~h}$; with a $2-\mathrm{h}$ incubation period, resolution of anatomic structures of interest and delineation of focal brain lesions were optimal. Adjacent sections were postfixed over paraformaldehyde vapor and stained with cresyl violet to assess tissue integrity.

Experimental protocols. Brains from animals that underwent right carotid artery ligation followed by $2.75 \mathrm{~h}$ of $8 \%$ $\mathrm{O}_{2}$ exposure were examined after a 1-h posthypoxia recovery period; in samples from eight animals, lesioned in two independent experiments, the patterns of changes in $\mathrm{Cy}$ tOx activity and Nissl staining observed in forebrain ipsilateral to ligation were similar. In contrast, in brains from animals examined $24 \mathrm{~h}$ posthypoxia $(n=22)$, there was considerable interexperiment variability in the extent of ipsilateral cerebral edema and disruption of CytOx activity and Nissl staining; animals from two experiments that yielded the most uniform, intermediate severity lesions were analyzed $(n=11)$. In addition, three animals from one of these litters were examined on PND 12, $5 \mathrm{~d}$ after lesioning.

To delineate the relationship between duration of hypoxic exposure and extent of disruption of CytOx activity, additional animals were exposed to $0(n=3), 1(n=$ $4)$, or $2(n=4)$ h of $8 \% \mathrm{O}_{2}$ after carotid ligation; they were killed after a 1-h recovery period in room air.

To determine whether the regional distribution of the acute changes in CytOx activity elicited by hypoxiaischemia represented a distinctive response to this pathophysiologic insult, we also assessed the acute changes in CytOx activity elicited by focal intracerebral injections of the neurotoxic excitatory amino acid agonist NMDA. In PND 7 ether-anesthetized rats, NMDA $(5 \mathrm{nmol} / 0.5 \mu \mathrm{L}$ saline, $n=4$ ) was injected through the right dorsal hippocampus into posterior striatum, using previously published methods (18), and the animals were killed by decapitation $1 \mathrm{~h}$ later.

Data analysis. The intensity of the reaction product of the CytOx histochemical assay closely correlates with CytOx activity measured spectrophotometrically, and the OD response is linear $(8,9)$. A computerized image analysis system (Imaging Research Inc., St. Catherines, Ontario, Canada) was used to measure OD bilaterally in CytOx-stained sections ( $\geq 5$ readings per section, $\geq 4$ sections per region per brain) with an arbitrary OD scale. OD 
values were obtained as point measurements from the darkest areas within each region of interest. Readings were obtained in nine subcortical structures: hippocampus CA1, $\mathrm{CA} 3, \mathrm{CA} 4$, upper blade of dentate gyrus, and lower blade of dentate gyrus; medial and lateral habenula; striatum; and posterolateral thalamic nucleus (which are identified in Fig. 1); for each brain, mean OD readings from these nine regions of each hemisphere were calculated and used for subsequent statistical analysis.

No quantitative data were obtained from cortex because we believed that several factors would limit the reliability of densitometry measurements in this region: the lesioned cortex was particularly susceptible to freezing artifact, edema was more prominent than in subcortical structures, and the distribution of cortical deficits in CytOx activity was often patchy (and thus particularly susceptible to sampling bias). No consistent columnar distribution in the pattern of loss of activity in cortex was discerned. Preliminary densitometric analysis of CytOx activity in brain sections from untreated PND 7-8 brain revealed uniform patterns, with the highest hippocampal activity in CA3 (as in adult brain); the distribution of CytOx activity in the contralateral (left) hemispheres of lesioned animals was the same as in untreated animals (data not shown). In each experiment, OD values in unlesioned tissue were within the same range.
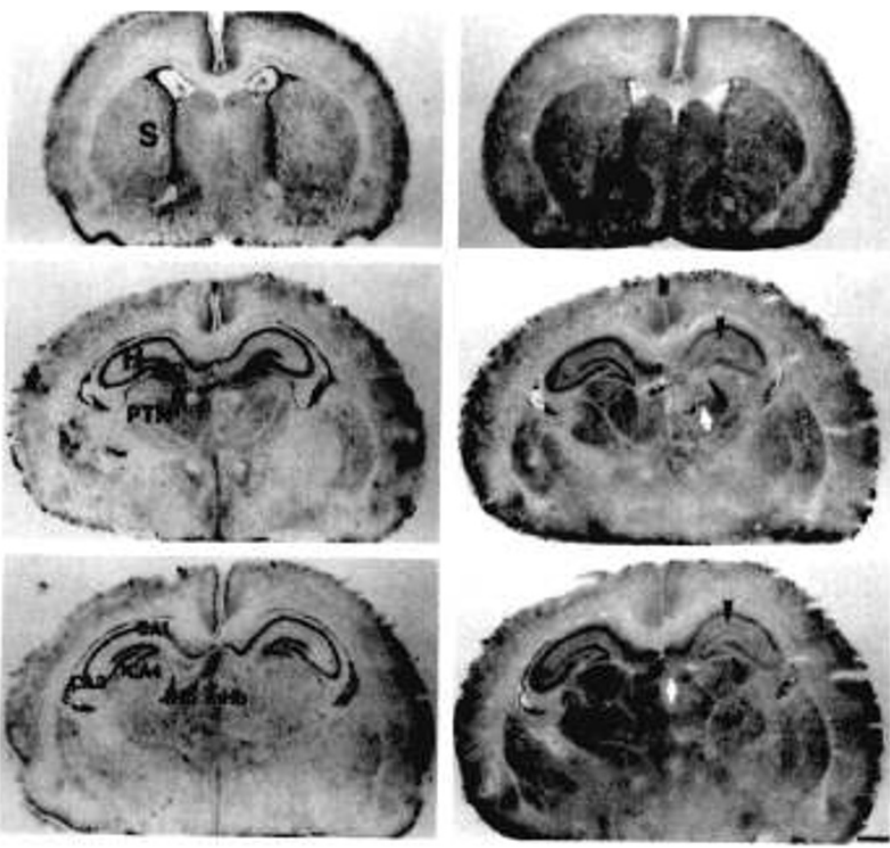

Figure 1. These coronal brain sections were prepared from a PND 7 rat that underwent right carotid artery ligation on PND 7, followed by exposure to $8 \% \mathrm{O}_{2}$ for $2.75 \mathrm{~h}$, and was killed $1 \mathrm{~h}$ later. Adjacent sections, at the levels of midstriatum (upper panels), posterolateral thalamic nucleus (middle panels), and habenula (lower panels), were stained for Nissl substance with cresyl violet (left) and for CytOx activity (right) (see Methods). There were no deficits in Nissl staining. Reductions in CytOx activity were readily apparent in habenula and posterolateral thalamic nucleus (white arrows) and dentate gyrus (black arrowheads) of the right hemisphere. $S$, striatum; $H$, hippocampus; $D G$, hippocampal dentate gyrus; $l H b$, lateral habenula; $m H b$, medial habenula; PTN, posterolateral thalamic nucleus. Scale bar $=1 \mathrm{~mm}$.
A microcomputer-based software program [Statview (19)] was used for descriptive statistics and calculation of analysis of variance (ANOVA). Values from the 1-h and 24-h posthypoxia animals were analyzed independently; in each group, regional and side-side differences in CytOx activity were compared by two-way ANOVA.

\section{RESULTS}

In animals examined $1 \mathrm{~h}$ after right carotid artery ligation and $2.75 \mathrm{~h}$ of $8 \% \mathrm{O}_{2}$ exposure (Figs. 1 and 2), loss of CytOx activity was readily apparent in the ipsilateral hippocampus in six of eight, whereas in the striatum and cortex, CytOx activity seemed intact. There were prominent reductions in CytOx activity in two other brain
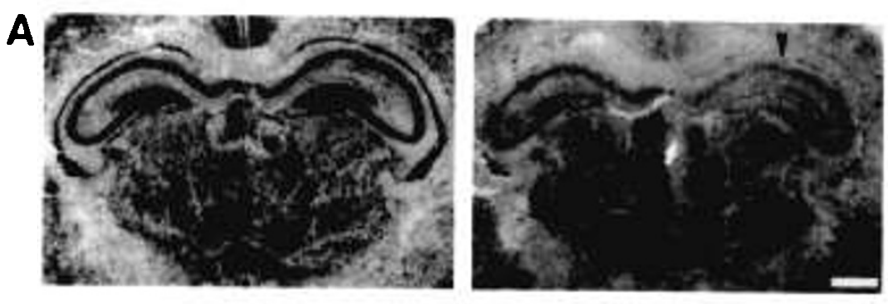

B
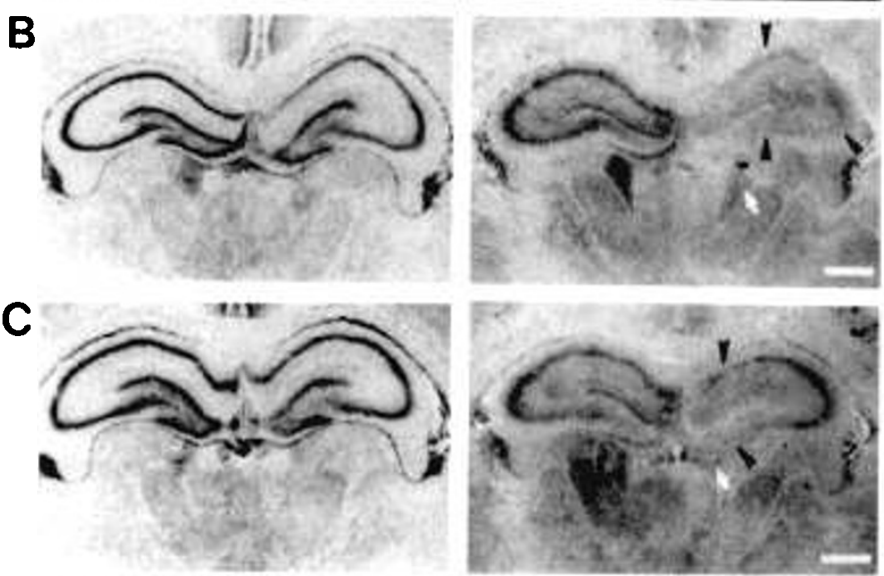

D
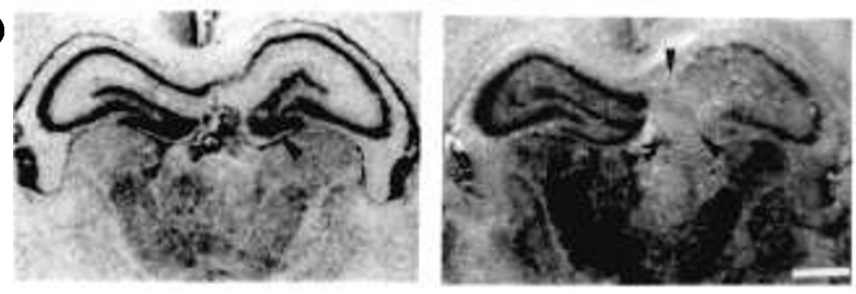

Figure 2. These coronal brain sections were prepared from four animals that underwent right carotid artery ligation on PND 7 followed by $2.75 \mathrm{~h}$ of $8 \% \mathrm{O}_{2}$ exposure and were killed $1 \mathrm{~h}$ later. Corresponding adjacent sections, at the level of midhippocampus, stained for Nissl substance (left) and CytOx activity (right) (see Methods) demonstrate the range of abnormalities detected in the hypoxic-ischemic hemisphere. Overall, loss of CytOx activity was more extensive than loss of Nissl staining. Panel $A$ demonstrates intact Nissl staining together with diffuse reductions in CytOx activity in the right hippocampus (black arrowheads) and habenula (white arrow). Panels $B$ and $C$ demonstrate subtle attenuation of hippocampal and thalamic Nissl staining and, in adjacent sections, diffuse loss of CytOx activity in the lesioned hippocampus (black arrowheads) and posterolateral thalamic nucleus (white arrow). Panel $D$ demonstrates the most severe abnormality in Nissl staining observed in this group of animals, in dentate gyrus (arrowhead), and an adjacent section in which no CytOx activity was discernible in dentate gyrus (arrowheads). Scale bars $=1 \mathrm{~mm}$. 
regions, the ipsilateral habenula (medial $>$ lateral) and the posterolateral thalamic nucleus.

Comparison of adjacent sections, assayed for CytOx activity and stained for Nissl substance, generally revealed disparate patterns. In contrast with the marked disruption of CytOx activity, there were only subtle focal reductions in Nissl staining in four of eight animals; in the other four, Nissl staining was normal. Figure 2 demonstrates the range of abnormalities encountered. Of note, in the lesioned hippocampus, reductions in CytOx activity were heterogeneously distributed and often were most pronounced in the dentate gyrus; generally, when $\mathrm{CO}$ activity was suppressed in the lesioned dentate gyrus there were coincident reductions in CytOx activity in the ipsilateral habenula and posterolateral thalamic nucleus. Features of the most severe histopathologic lesion in this group included distortion and reduced Nissl staining in the dentate gyrus and habenula (Fig. $2 D$ ). In the brains that demonstrated abnormalities in Nissl staining, comparison of multiple serial sections revealed that disruption of CytOx activity extended over a considerably greater area than did corresponding alterations in Nissl staining.

Figure 3 compares CytOx activity (measured by densitometry) in nine regions of the ischemic and contralateral hemispheres of animals analyzed 1 or $24 \mathrm{~h}$ after right carotid artery ligation and $2.75 \mathrm{~h}$ of $8 \% \mathrm{O}_{2}$ exposure; trends were similar at the two time intervals. At $1 \mathrm{~h}$ posthypoxia, reductions in CytOx activity in the lesioned hemisphere ranged from $10 \%$ in striatum to $42 \%$ in the posterolateral thalamic nucleus. There was no predictable relationship between level of intrinsic CytOx activity and severity of CytOx deficits incurred; for example, in CA3, with the highest intrinsic activity, intermediate reductions were observed. Two-way ANOVA, considering regional and side-side differences, revealed significant differences for both factors $(p=0.0001)$, with a significant interaction between these variables $(p=0.007)$. Within hippocampus, although deficits in the five regions compared were in a similar range, there were significant interregion differences ( $p=0.0001$, two-way ANOVA). In striatum, CytOx activity seemed to be relatively preserved; yet, if the striatum was arbitrarily bisected in a horizontal plane and CytOx activity in the upper and lower halves were compared, CytOx activity was reduced by $18 \pm 2 \%$ (mean \pm SEM) in the upper half, compared with a $9 \pm 1 \%$ reduction in the lower half $(p<$ 0.001 , paired $t$ test). Generally, with this lesioning method, irreversible striatal injury is most pronounced dorsally.

Figure 4 compares hippocampal CytOx activity in animals that underwent right carotid ligation on PND 7 and were exposed to $8 \% \mathrm{O}_{2}$ for $0 \mathrm{~h}(A), 1 \mathrm{~h}(B), 2 \mathrm{~h}(C)$, and $2.75 \mathrm{~h}(D)$ and were killed $1 \mathrm{~h}$ later and demonstrates the progression of ipsilateral deficits in CytOx activity. In three of three animals that underwent ligation without subsequent hypoxia, CytOx activity was unchanged. In animals exposed to $8 \% \mathrm{O}_{2}$ for $1 \mathrm{~h}$, diffuse reductions in

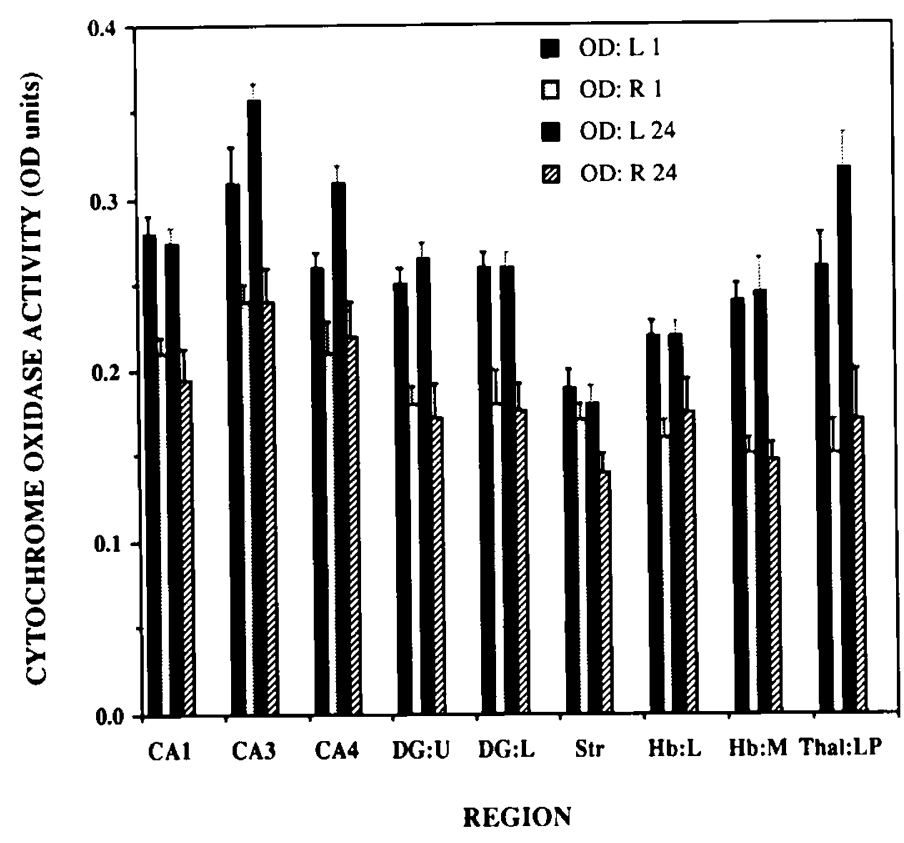

Figure 3. This graph compares CytOx activity in brain sections prepared from PND 7 rats that underwent right carotid ligation followed by $2.75 \mathrm{~h}$ of $8 \% \mathrm{O}_{2}$ exposure and were killed $\mathrm{l}(n=8)$ or $24 \mathrm{~h}(n=11)$ later. CytOx activity was assayed in frozen brain sections (see Methods), and OD was measured bilaterally in nine brain regions by computerized densitometry (based on $\geq 5$ readings per section and $\geq 4$ sections per region per brain). Because $\mathrm{OD}$ is directly and linearly related to CytOx activity, values (means \pm SEM) were expressed as arbitrary OD units." $O D: L l$ and $O D: R l$ indicate values from left and right hemispheres of animals examined $1 \mathrm{~h}$ posthypoxia; $O D: L 24$ and $O D: R 24$ indicate values from left and right hemispheres of animals examined $24 \mathrm{~h}$ posthypoxia. $D G: U$, upper blade of dentate gyrus; $D G: L$, lower blade of dentate gyrus; Str, striatum; $H b: L$, lateral habenula; $H b: M$, medial habenula; Thal:LP, posterolateral thalamic nucleus.

hippocampal CytOx activity were apparent in two of four animals and CytOx activity was minimally $(<10 \%$ by densitometry) and inconsistently reduced in the ischemic hemisphere in the other two brains. After $2 \mathrm{~h}$ of $8 \% \mathrm{O}_{2}$ exposure, there were extensive ipsilateral reductions in CytOx activity in four of four animals.

To determine whether the heterogeneous pattern of reductions in hippocampal CytOx activity was a distinctive response to hypoxia-ischemia, the effects of intracerebral injections of the neurotoxin NMDA on CytOx activity were assessed. Posterior striatal injection of 5 nmol of NMDA in PND 7 rats typically elicits minimal focal neuronal injury in overlying dorsal hippocampus, with pyramidal cell loss adjacent to the needle track; $1 \mathrm{~h}$ postinjection, in four of four animals evaluated, there was diffuse suppression of hippocampal CytOx activity with no predilection for dentate gyrus, and adjacent Nisslstained sections were normal (not shown).

In animals examined $24 \mathrm{~h}$ after carotid ligation and 2.75 $\mathrm{h}$ of $8 \% \mathrm{O}_{2}$ exposure, overall there was considerable variation in the severity and distribution of tissue injury (as has been noted previously in this model). In each brain, there was a close anatomic correspondence between loss of CytOx activity and reduced Nissl staining in the lesioned hemisphere; Figure 5 demonstrates the 

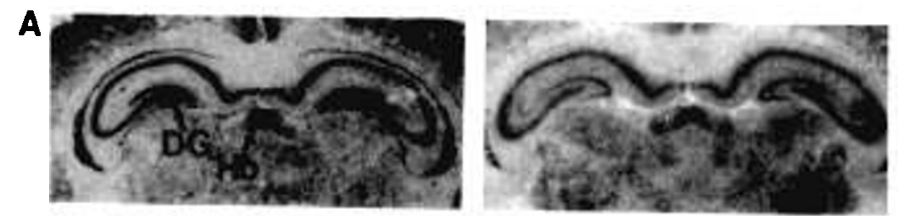

B
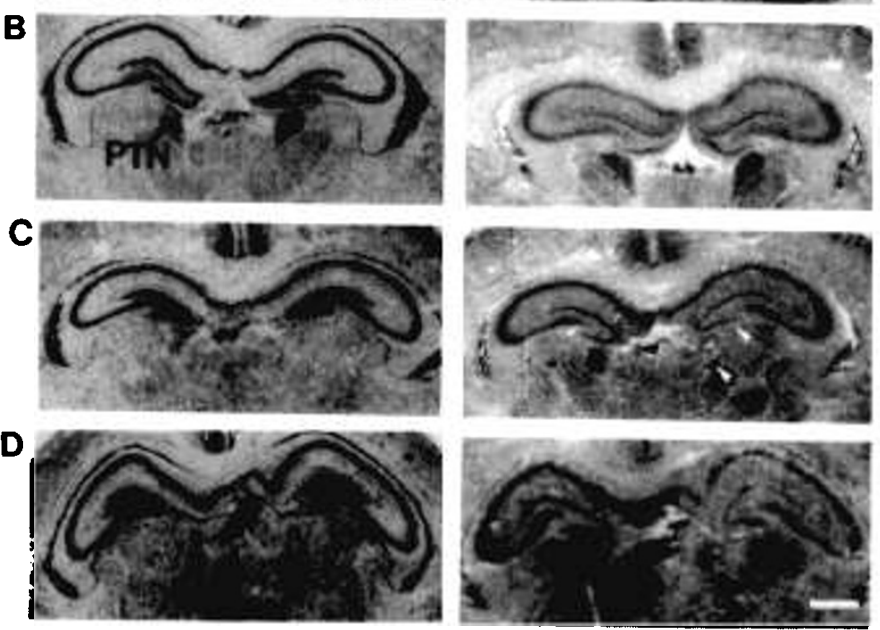

Figure 4. These coronal brain sections were prepared from animals that underwent right carotid ligation on PND 7 and were subsequently exposed to $8 \% \mathrm{O}_{2}$ for $0 \mathrm{~h}(A), 1 \mathrm{~h}(B), 2 \mathrm{~h}(C)$, and $2.75 \mathrm{~h}(D)$; all animals were killed after a $1-\mathrm{h}$ recovery period in room air. Corresponding adjacent brain sections, at the level of hippocampus, were stained for Nissl substance (left) and CytOx activity (right) (see Methods). No abnormalities of Nissl staining or suppression of CytOx activity were apparent in three of three animals that underwent right carotid ligation alone $(A)$. Of four animals exposed to $8 \% \mathrm{O}_{2}$ for $1 \mathrm{~h}, \mathrm{CO}$ activity was minimally ( $<10 \%$ by densitometry) and inconsistently reduced in two $(B)$, and diffuse reductions in hippocampal CytOx activity were apparent in the other two brains. Subtle reductions in CytOx activity in the dentate gyrus $(D G)$ and posterior thalamic nucleus $(P T N)$ were detectable after $2 \mathrm{~h}$ of hypoxia $(C)$ (indicated by arrows). More widespread deficits in CytOx activity in hippocampus and habenula $(\mathrm{Hb})$ and subtle alterations in hippocampal Nissl staining were apparent after $2.75 \mathrm{~h}$ of $8 \% \mathrm{O}_{2}$ exposure $(D$; sections from same brain as presented in Fig. $2 D)$. Scale bar $=1 \mathrm{~mm}$.

range of abnormalities observed. Sections in panel $A$ have corresponding subtle deficits in lesioned striatum and hippocampus. Sections in panel $B$ demonstrate diffuse ipsilateral edema; in panel $B$, loss of Nissl staining and CytOx activity are most pronounced in dentate gyrus and thalamus. In both brains, reductions in CytOx activity in the ipsilateral cortex are also apparent, but interpretation of these deficits was confounded by cortical edema. Computerized densitometry confirmed widespread reductions in CytOx activity in the lesioned hemisphere, with a broad range in the magnitude of the deficits among brain regions analyzed (Fig. 3; $n=11$, two-way ANOVA, $p=0.0001$ ).

In three animals examined $5 \mathrm{~d}$ after lesioning, ipsilateral reductions in CytOx activity corresponded directly with the distribution of injury. Figure 6 demonstrates a representative, moderate-severity, unilateral forebrain lesion with striatal, hippocampal, and cortical atrophy. In the lesioned hemisphere, reductions in CytOx activity, most readily apparent in hippocampus and medial habenula, correspond closely with reductions in Nissl staining and substance loss; in the atrophic striatum, CytOx activity seems proportionate to tissue mass. There is no increase in CytOx activity in a nonneuronal distribution; thus, there is no evidence to suggest that reactive glia contribute substantially to measurable CytOx activity.

\section{DISCUSSION}

Focal reductions in CytOx activity provide one of the earliest detectable indicators of neuronal injury in this perinatal rodent stroke model. These acute reductions could reflect ischemia-induced suppression of CytOx functional activity, mitochondrial dysfunction, or plasma membrane injury in discrete neuronal populations.

Mitochondrial failure represents a critical step in the progression of irreversible ischemic neuronal injury $(2-4$, 6 ); it is interesting to consider that some inherited mitochondrial encephalopathies are characterized by recurrent strokes (20). In experimental stroke models in adult brain, functional suppression of mitochondrial activity precedes evidence of cell damage in selectively vulnerable regions (2), and mitochondria isolated from ischemiasusceptible and ischemia-resistant brain regions demonstrate different postischemia responses (3).

Whether the acute focal reductions in CytOx activity we observed in hypoxic-ischemic brain represent inevitable antecedents of cell death is unknown. This issue could not be addressed directly, because each animal could only be examined at one time point, and inferences based on population data were somewhat limited by the variability in the distribution and severity of histopathology. Overall, the distribution of early loss of CytOx activity and the distribution of deficits at subsequent time intervals 1-5 d later were similar. Yet, several findings suggest that early suppression of CytOx activity does not inevitably predict cell death. The reductions in CytOx activity were more uniform in the acute posthypoxic period than at $24 \mathrm{~h}$ posthypoxia. Although the temporal threshold to elicit irreversible tissue injury in this perinatal stroke model cannot be precisely defined, the minimum duration of exposure to elicit injury in a significant proportion of animals lies between 1 and $2 \mathrm{~h}$, and severity of injury increases with duration of hypoxia [and concurrent progressive ischemia (21)]. In all animals exposed to the threshold duration of hypoxia to elicit injury $(2 \mathrm{~h})$, there was prominent focal suppression of CytOx activity, whereas tissue injury after this duration of hypoxia is elicited less consistently. Thus, the threshold duration of hypoxic exposure to disrupt CytOx activity either slightly preceded or coincided with the threshold to elicit irreversible injury.

Histochemical staining with tetrazolium salts, which serve as markers of mitochondrial oxidative enzyme function, has also been used to delineate infarcted brain $(22,23)$. Cole et al. (23) compared the magnitudes of deficits in 2,3,5-triphenyltetrazolium chloride staining in brains of adult animals examined after $3 \mathrm{~h}$ of middle cerebral artery occlusion and in animals that underwent 
A
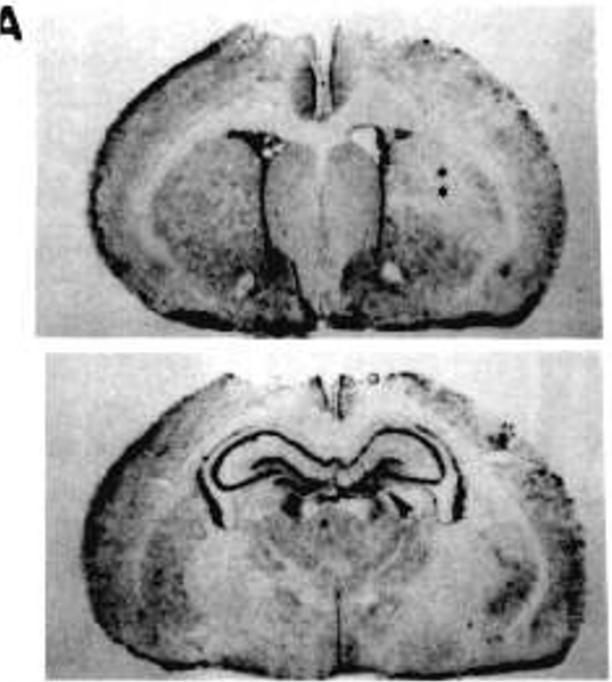

B

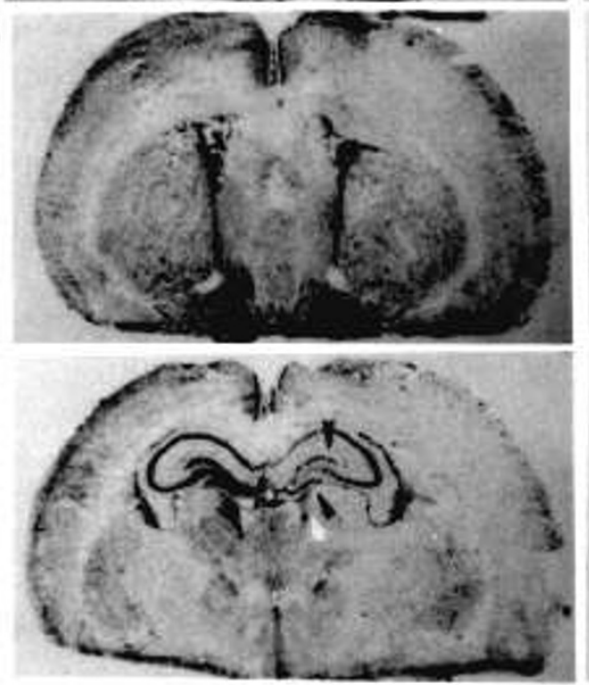

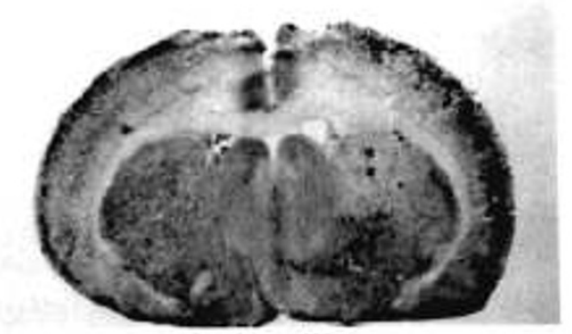
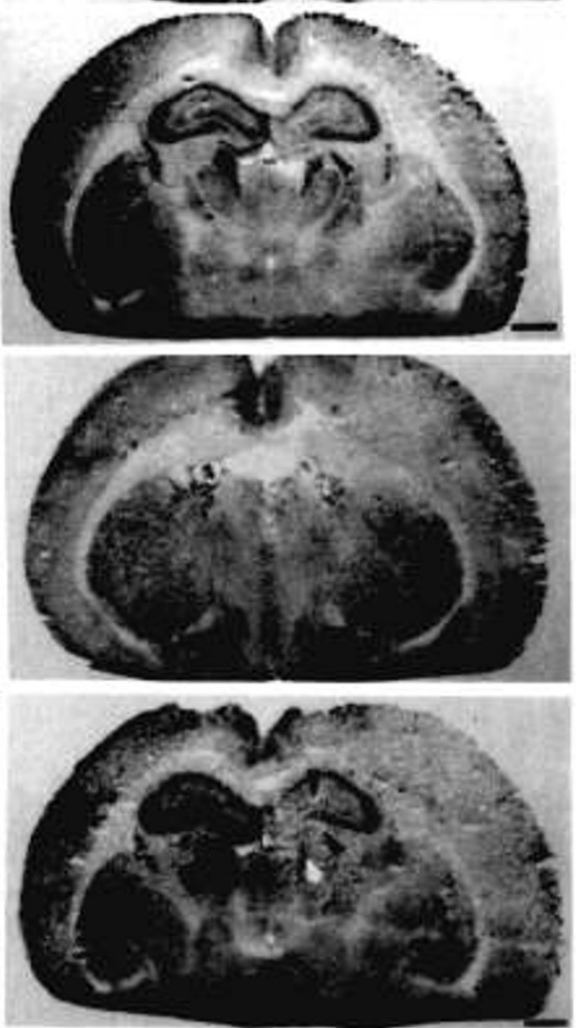

Figure 5. These coronal brain sections were prepared from two animals that underwent right carotid artery ligation on PND 7 , followed by 2.75 $\mathrm{h}$ of exposure to $8 \% \mathrm{O}_{2}$, and were killed $24 \mathrm{~h}$ later. They demonstrate the variability in the distribution and severity of deficits in the lesioned hemisphere apparent at this time interval posthypoxia. Adjacent sections, at the levels of midstriatum and hippocampus, were stained for Nissl substance (left) and CytOx activity (right) (see Methods). In each brain, there was a close correspondence between loss of Nissl staining and suppression of CytOx activity in the lesioned hemisphere. Sections in $A$ demonstrate subtle abnormalities in striatum (identified by asterisks) and hippocampus (arrowheads). Sections in $B$ demonstrate diffuse ipsilateral edema; loss of Nissl staining and CytOx activity was most pronounced in dentate gyrus (arrowheads) and thalamus (white arrow). Scale bars $=1 \mathrm{~mm}$.

the same procedure followed by a 2-h reperfusion period before they were killed; in the reperfusion group, the area of injury (identified histochemically) was reduced considerably, suggesting that the initial deficits did not inevitably predict irreversible injury.

Similarly, the preliminary NMDA lesioning data suggest that loss of CytOx activity is not necessarily indicative of irreversible injury; the diffuse suppression of CytOx activity in the injected hippocampus was much more widespread than the distribution of irreversible neuronal injury resulting after injection of the same dose of NMDA (5 nmol) (24).

Our data also suggest that factors contributing to intrinsic vulnerability, biochemical antecedents, and timing of irreversible ischemic injury in the developing brain may differ among specific neuronal populations. The heterogeneous acute suppression of CytOx activity within hippocampus cannot be attributed to vascular anatomy, metabolic activity or distribution of excitatory amino acids receptors (11). The distinctive early reductions in CytOx activity in the dentate gyrus are intriguing both because this region seems particularly susceptible to ischemic injury in the immature brain (as compared with adult animals) and because the pattern seems to be a distinctive response to acute ischemia (compared with the effects of NMDA lesioning). Yet, it is also important to consider that the distribution of ischemic hippocampal injury is typically widespread.

Findings in the striatum also suggest that the antecedents of irreversible injury may vary among brain regions. Even though regional analysis did demonstrate that acute reductions in CytOx activity were more pronounced in the more vulnerable dorsal striatum, the acute reductions in CytOx activity were relatively mild in comparison with 

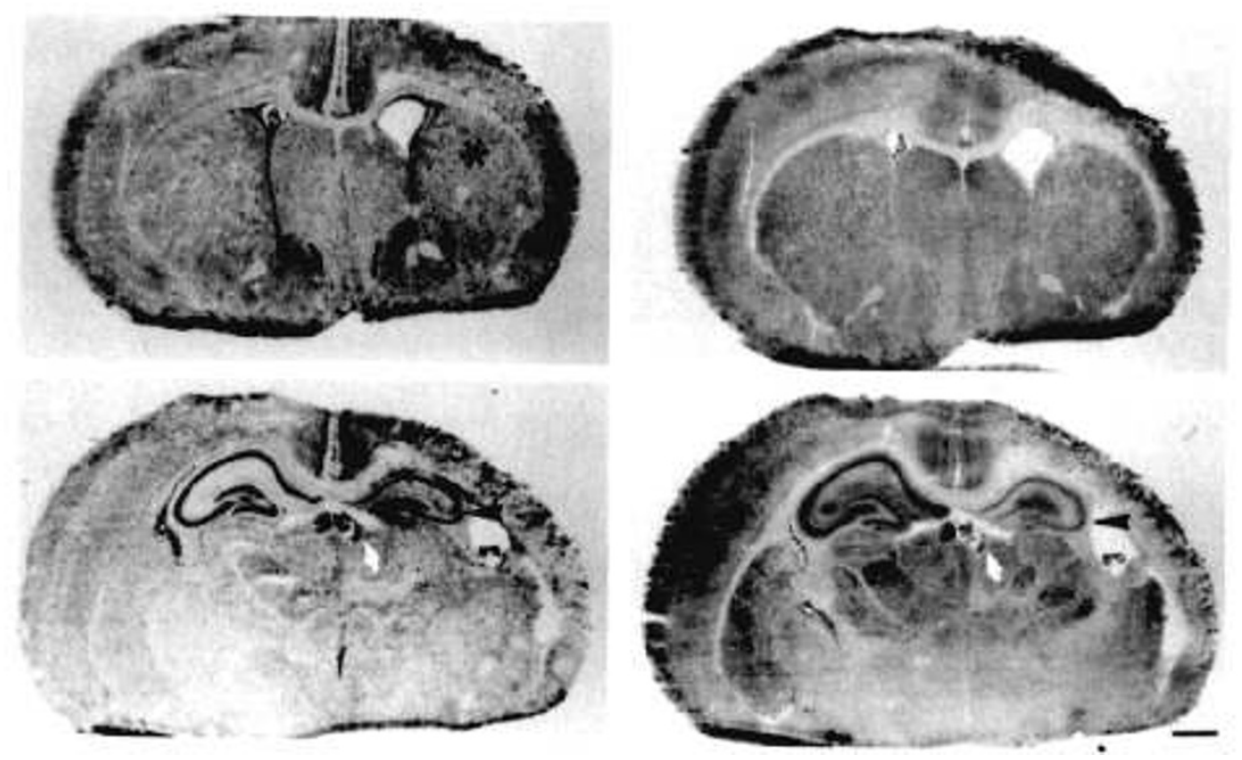

Figure 6. These coronal brain sections were prepared from an animal that underwent right carotid artery ligation on PND 7 , followed by $2.75 \mathrm{~h}$ of $8 \% \mathrm{O}_{2}$ exposure, and was killed $5 \mathrm{~d}$ later. Adjacent sections, at the levels of midstriatum and habenula, stained for Nissl substance (left) and CytOx activity (right) demonstrate the features of irreversible injury. In the lesioned hemisphere, there is diffuse striatal atrophy (identified by an asterisk) with proportionate preservation of CytOx activity. Loss of CytOx activity in hippocampus (black arrowheads) and habenula (white arrows) corresponds closely with reductions in Nissl staining and substance loss. Scale bar $=1 \mathrm{~mm}$.

findings in the ipsilateral hippocampus and in relation to the extent of irreversible injury that typically evolves in this region $(10,13)$. It is conceivable that the relatively lower density of neurons (which are the major source of CytOx activity) in striatum accounts for some of the discrepancies; alternatively, the results might suggest that onset of injury in striatum is relatively delayed compared with other vulnerable regions.

The prominent early reductions in CytOx activity in periventricular structures such as the habenula and dorsal thalamic nucleus, in addition to the dentate gyrus, were unexpected. These regions do not share either particularly high intrinsic oxidative activity or high densities of excitatory amino acid receptors (11). There are no data to suggest that these vulnerable regions share any tissuespecific isozymes of CytOx or have lower concentrations of mitochondria per neuron than less affected regions. Although a periventricular distribution might suggest exposure to a cerebrospinal fluid-transmitted toxin, this mechanism cannot fully explain our findings, because effects were restricted to the ischemic hemisphere. Of interest, in studies of a related model in which perinatal stroke was induced by carotid ligation followed by hypobaric exposure, Ikonomidou et al. (25) found, by light and electron microscopy, that the highest incidence of acute vacuolar changes with degeneration of neuronal dendrites and cell bodies was in medial habenula (94\%), dentate gyrus $(81 \%)$, and caudate $(71 \%)$; CA1 and CA3 were much less commonly affected. In injured nerve cells, the earliest evidence of degeneration they identified was in mitochondria; within the same brain, they noted that distinctive morphologic stages of mitochondrial degeneration could be identified concurrently in different regions. They emphasized the morphologic similarities to the cytopathology of excitotoxic injury but could provide no explanation for the anatomic distribution of acute injury. Their findings may well reflect the same underlying pathophysiologic mechanisms that contribute to the prominent early loss of CytOx activity in these structures after hypoxia-ischemia.

In this perinatal stroke model, considerable data suggest that excitatory amino acids contribute to the pathogenesis of irreversible neuronal injury. In vivo microdialysis studies demonstrate increases in glutamate efflux in striatum and hippocampus during the evolution of injury $(26,27)$ and systemic administration of the noncompetitive NMDA antagonist MK-801 is neuroprotective (14). In vitro observations suggest that metabolically compromised neurons are more susceptible to excitatory amino acid-mediated injury (28). Thus, in ischemic brain, neurons in which mitochondria are more readily damaged or in which mitochondrial recovery mechanisms are less effective, which are identified by loss of CytOx activity, may be most vulnerable to excitatory amino acidinduced damage. Of note, there is increasing evidence to suggest that similar mechanisms may play a pivotal role in the pathogenesis of several chronic neurodegenerative disorders (29).

The clinical utility of near-infrared spectroscopy to monitor cerebral CytOx activity in vivo in neonates at risk for cerebral ischemia is currently being evaluated (30); our results suggest that this approach may yield important new information about the mechanism and timing of ischemic brain injury in neonates.

Histochemical analysis of CytOx activity during the evolution of ischemic injury represents a sensitive anatomic method for delineation of differences in susceptibility to and timing of ischemic injury among discrete 
neuronal populations. This assay may also facilitate evaluation of the efficacy of neuroprotective interventions aimed at preserving or restoring neuronal energy metabolism in experimental stroke models.

Acknowledgments. The authors thank Dr. Roger Albin for introducing the cytochrome oxidase assay to us.

\section{REFERENCES}

1. Hillered L, Siesjo BK, Arfors KE 1984 Mitochondrial response to transien forebrain ischemia and recirculation in the rat. J Cereb Blood Flow Metab $4: 438-446$

2. Sims NR, Pulsinelli WA 1987 Altered mitochondrial respiration in selectively vulnerable brain regions following transient forebrain ischemia in the rat. J Neurochem 49:1367-1374

3. Sims N 1991 Selective impairment of respiration in mitochondria isolated from brain subregions following transient forebrain ischemia in the rat. J Neurochem 56:1836-1844

4. Nakahara I, Kikuchi H, Taki W, Nishi S, Kito M, Yonekawa Y, Goto Y, Ogata N 1991 Degradation of mitochondrial phospholipids during experimental cerebral ischemia in rats. J Neurochem 57:839-844

5. Dimlich R, Showers MJ, Shipley MT 1990) Densitometric analysis of cytochrome oxidase in ischemic rat brain. Brain Res 516:181-191

6. Wagner KR, Kleinholz M, Myers RE 1990 Delayed onset of neurologic deterioration following anoxia/ischemia coincides with appearance of impaired brain mitochondrial respiration and decreased cytochrome oxidase activity. J Cereb Blood Flow Metab 10:417-423

7. Hevner RF, Wong-Riley MT 1989 Brain cytochrome oxidase: purification antibody production, and immunohistochemical/histochemical correlations in the CNS. J Neurosci 9:3884-3898

8. Wong-Riley MT 1989 Cytochrome oxidase: an endogenous metabolic marker for neuronal activity. Trends Neurosci 12:94-101

9. Gonzales-Lima F, Garrosa M 1991 Quantitative histochemistry of cytochrome oxidase in rat brain. Neurosci Lett 123:251-253

10. Rice JE, Vannucci RC, Brierley JB 1981 The influence of immaturity on hypoxic-ischemic brain damage in the rat. Ann Neurol 9:131-141

11. Silverstein FS, Torke L, Barks J, Johnston MV 1987 Hypoxia-ischemia produces focal disruption of glutamate receptors in developing brain. Dev Brain Res 34:33-39

12. Wasterlain CG, Hattori H, Yang C, Schwartz PH, Fujikawa DG, Morin AM, Dwyer BE 1990 Selective vulnerability of neuronal subpopulations during ontogeny reflects discrete molecular events associated with normal brain development. In: Wasterlain CG, Vert P (eds) Neonatal Seizures. Raven Press, New York, pp 69-81
13. Silverstein FS, Johnston MV 1984 Effects of hypoxia-ischemia on monoamine metabolism in the immature brain. Ann Neurol 15:342-347

14. McDonald JW, Silverstein FS, Johnston MV 1987 MK-801 protects the neonatal brain from hypoxic-ischemic damage. Eur J Pharmacol 140:359-361

15. Palmer C, Brucklacher RM, Christensen MA, Vannucci RC 1990 Carbohydrate and energy metabolism during the evolution of hypoxic-ischemic brain damage in the immature rat. J Cereb Blood Flow Metab 10:227-235

16. Towfighi J, Yager JY, Housman C, Vannucci RC 1991 Neuropathology of remote hypoxic-ischemic damage in the immature rat. Acta Neuropathol (Berl) 81:578-587

17. Vannucci RC 1990 Experimental biology of cerebral hypoxia-ischemia: relation to perinatal brain damage. Pediatr Res 27:317-326

18. McDonald JW, Roeser NF, Silverstein FS, Johnston MV 1989 Quantitative assessment of neuroprotection against NMDA-induced brain injury. Exp Neurol 106:289-296

19. Feldman DS, Gagnon J, Hofmann R, Simpson J 1988 Statview II. Abacus Concepts Inc, Berkeley, CA

20. Pavlakis SG, Phillips PC, DiMauro S, De Vivo DC, Rowland LP 1984 Mitochondrial myopathy, encephalopathy, lactic acidosis, and strokelike episodes: a distinctive clinical syndrome. Ann Neurol 16:481-488

21. Silverstein FS, Buchanan K, Johnston MV 1984 Pathogenesis of hypoxic ischemic brain injury in a perinatal rodent model. Neurosci Lett 49:271-278

22. Liszczak TM, Hedley-Whyte ET, Adams JF, Han DH, Kolluri VS, Vacanti FX, Heros RC, Zervas NT 1984 Limitations of tetrazolium salts in delineating infarcted brain. Acta Neuropathol (Berl) 656:150-157

23. Cole DJ, Drumond JC, Ghazal EA, Shapiro HM 1990 A reversible component of cerebral injury as identified by the histochemical stain 2,3,5-triphenyltetrazolium chloride (TTC). Acta Neuropathol (Berl) 80:152-155

24. Barks JD, Nair MPN, Schwartz SA, Silverstein FS 1993 Potentiation of $\mathrm{N}$-methyl-D-aspartate-mediated brain injury by a human immunodeficiency virus-1-derived peptide in perinatal rodents. Pediatr Res 34:192-198

25. Ikonomidou C, Price MT, Mosinger JL, Frierdich G, Labruyere J, Olney J 1989 Hypobaric-ischemic conditions produce glutamate-like cytopathology in infant rat brain. J Neurosci 9:1693-1700

26. Gordon K, Simpson J, Statman D, Silverstein FS 1991 Effects of perinatal stroke on striatal amino acid efflux in rat studies with in vivo microdialysis. Stroke 22:928-932

27. Silverstein FS, Naik B, Simpson J 1991 Hypoxia-ischemia stimulates hippocampal glutamate efflux in perinatal rat brain: an in vivo microdialysis study. Pediatr Res 30:587-590

28. Novelli A, Reilly JA, Lysko PG, Henneberry RC 1988 Glutamate becomes neurotoxic via the $\mathrm{N}$-methyl-D-aspartate receptor when intracellular energy levels are reduced. Brain Res 451:205-212

29. Beal MF, Hyman BT, Koroshetz W 1993 Do defects in mitochondrial energy metabolism underlie the pathology of neurodegenerative diseases? Trends Neurosci 16:125-131

30. Hirtz DG 1993 Report of the National Institute of Neurological Disorders and Stroke Workshop on near infrared spectroscopy. Pediatrics 21:414-417 